\section{Cahiers de Narratologie}

Analyse et théorie narratives

$10.2 \mid 2001$

La voix narrative

\title{
Le discours indirect libre dans le récit de fiction
}

\author{
María Muñoz Romero
}

\section{OpenEdition \\ Journals}

Édition électronique

URL : http://journals.openedition.org/narratologie/10235

DOI : 10.4000/narratologie.10235

ISSN : 1765-307X

Éditeur

LIRCES

Édition imprimée

Date de publication : 1 janvier 2001

Pagination : 285-294

ISBN : 2914561032

ISSN : 0993-8516

Référence électronique

María Muñoz Romero, "Le discours indirect libre dans le récit de fiction », Cahiers de Narratologie [En ligne], 10.2 | 2001, mis en ligne le 01 janvier 2001, consulté le 11 juin 2020. URL : http://

journals.openedition.org/narratologie/10235; DOI : https://doi.org/10.4000/narratologie.10235 


\title{
LE DISCOURS INDIRECT LIBRE DANS LE RÉCIT DE FICTION
}

\author{
María MUÑOZ ROMERO \\ Université de Séville
}

Depuis qu'en 1887 elle fut découverte par Adolf Tobler dans l'œuvre de Zola, cette stratégie de reproduction discursive, qui connut son apogée dans le réalisme psychologique de la seconde moitié du XIXème siècle, n'a cessé de fasciner les linguistes et les critiques littéraires qui se sont interrogés tout particulièrement sur sa date d'apparition ${ }^{1}$ et sur son origine $^{2}$.

${ }^{1}$ Pour quelques-uns, cette forme remonte au Moyen Âge, et ils trouvent déjà dans des textes médiévaux des fragments avec ces particularités (cf. B. Cerquiglini : "Le style indirect libre et la modernité ", Langages, 73 (1984), 7-16). D'autres, cependant, pensent que ces manifestations médiévales n'obéissent pas à une véritable conscience stylistique, et attribuent ces usages à l'absence d'une syntaxe claire et solide. Par conséquent, les exemples rencontrés n'obéiraient pas à un choix délibéré. Il s'agirait d'exemples confus dûs à la syntaxe imprécise et ambiguë de l'époque. Pour ceux qui pensent de cette façon, le DIL appartient clairement à la littérature moderne, et ils n'attribuent ce style qu'à des auteurs contemporains (cf. A. Banfield : "Où l'épistémologie, le style et la grammaire rencontrent l'histoire littéraire : le développement de la parole et de la pensée représentées ", Langue française, 44 (1979), 9-26). Finalement, d'autres admettent l'existence du DIL au Moyen Âge, bien qu'ils considèrent que le vrai développement de ce procédé a lieu à partir du romantisme. Ainsi Bally, qui résume en ces termes la trajectoire historique discontinue de cette stratégie : "Connu de l'ancien français, le style indirect libre meurt, ou peu s'en faut, à la Renaissance, si ce n'est avant; il ne survit que chez les gardiens de la libre tradition gauloise; Rabelais en présente des traces. La Fontaine en fait un de ses procédés favoris, et avec quel charme incomparable il le manie ! Les purs classiques l'ignorent, asservis qu'ils sont à la phrase latine, à laquelle ce tour est étranger. Il reparaît chez les émancipateurs ; Rousseau le pratique spontanément, les romantiques le remettent à la 
Le DIL constitue une stratégie selon laquelle sont reproduits les contenus d'une conscience - pensées, perceptions, sentiments, mots - dans leur propre espace et dans leur propre temps, mais par la voix du narrateur. L'expérience du personnage est actualisée par le narrateur, qui adopte en partie son système déictique et imite ses modes de dire, de telle sorte qu'il se produit une confluence entre le point de vue du narrateur et celui du personnage. Cette confluence se manifeste en surface du texte par la superposition de deux situations d'énonciation - celle du narrateur et celle de l'actant-, ce qui se traduit par la coprésence de marques linguistiques d'origines différentes.

Certes, nous trouvons dans le DIL des éléments généralement considérés incompatibles : d'une part, il présente la dissociation de deux actes d'énonciation, et de ce fait le caractère mimétique du DD, et d'autre part, la perte d'autonomie des déictiques personnels et des temps verbaux du discours cité, caractéristique du DI. En effet, les traits syntaxiques qui permettent de reconnaître un DIL sont assez particuliers : d'un côté, la troisième personne est corréférentielle à celui qui parle, pense ou ressent, au lieu de la première personne ; d'un autre côté, les adverbes déictiques, centrés sur le «ici » et le «maintenant » du personnage, coexistent avec l'imparfait, le plus-que-parfait et le conditionnel, temps inactuels qui ont leur point de repère dans le temps du récit ${ }^{3}$,

mode et chez Flaubert il devient une forme d'art capable des effets les plus délicats » («La pensée et la Langue (de M. F. Brunot) ", Bulletin de la Société linguistique de Paris, Paris : Champion, 1922).

2 S'agit-il d'un procédé réservé à la narration littéraire ou relève-til également de l'usage ordinaire de la langue ? Provient-il réellement d'une perte de la conjonction qui introduit le DI ou dérive-t-il de la tendance que manifeste la langue littéraire à s'approcher de la langue parlée et à présenter sans grands artifices syntaxiques les expressions quotidiennes des personnages? Ce sont des questions qui n'ont toujours pas de réponses définitives, après des années de discussions, et que, pour des raisons d'espace, nous n'aborderons pas ici.

${ }^{3}$ Bien que ce ne soit pas très fréquent, la possibilité d'utiliser les temps du discours (actuels) et de les alterner éventuellement avec ceux du récit (inactuels) existe pour le DIL. Dans ce dernier cas, on pourrait parler de repérage temporel mixte. 
raison pour laquelle le lecteur a l'impression de se trouver à la fois dans le «maintenant » du personnage et dans le « alors » du récit.

Ainsi, la subordination du discours cité au discours citant n'est que partielle, car elle permet que quelques marques énonciatives - déictiques spatiaux et temporels - et toutes les marques d'expressivité du discours original - interrogations, exclamations, modalités appréciatives, phrases inachevées, phrases nominales, etc. - se maintiennent intactes.

De cette façon, on perçoit toujours, d'une manière immédiate, la voix du personnage-énonciateur à travers un narrateur presque complètement effacé. Le narrateur se situe à la place du personnage, dans son intérieur, et s'exprime selon son point de vue. On rapporte les mots, les pensées et les perceptions du personnage en les recréant dans sa propre conscience. Nous avons ainsi l'impression que le personnage parle, pense, ressent ou perçoit avec ses propres mots.

En effet, dans le DIL, nous observons un procédé inverse à celui présenté dans le DI : au lieu de transposer l'énonciation du locuteur cité à sa propre énonciation, le narrateur qui cite au style indirect libre se transpose au "ici » et au «maintenant» de son personnage, et tente de reproduire sa façon de dire, de penser ou de percevoir. On n'attribue pas seulement des contenus, comme dans le DI, mais aussi des mots: on rapporte des sentiments, des pensées, des perceptions, en imitant leur discours imaginaire. Cependant, à la différence du DD, la mimesis ne peut être complète : l'apparition de la troisième personne et des temps inactuels dénonce, ainsi que nous l'avancions, la présence du narrateur qui parle ou pense ou ressent avec le personnage.

Lorsque le «alors » du récit s'unit au «maintenant »du personnage, la confluence de points de vue se perçoit, en effet, comme confluence de voix ${ }^{4}$. Ainsi, tandis que dans le DI

${ }^{4}$ L'utilisation de l'imparfait met en évidence cette confluence : en effet, la valeur aspectuelle imperfective de ce temps, qui présente l'action en cours, sans faire référence ni à son début ni à sa fin, le convertit en la forme verbale appropriée pour indiquer, sans interrompre le récit, la confluence du passé du narrateur et du présent du personnage (cf. G. Reyes : Polifonía textual, Madrid: Gredos, 1984 , p. 84). 
nous entendons une seule voix, celle du narrateur, et dans le DD deux voix juxtaposées, parfaitement délimitées, dans le DIL nous percevons aussi deux voix - et non seulement deux points de vue -, mais complètement imbriquées, fondues, représentées en surface par la superposition de deux systèmes référentiels distincts et incompatibles.

On comprend facilement la confusion des grammairiens devant une forme de citation qui ne peut être attribuée ni entièrement au narrateur ni entièrement au personnage. Il s'agit d'un mode d'énonciation basé sur la polyphonie, où l'on entend deux voix intimement liées, celle du narrateur et celle du personnage. Le lecteur découvre cette dualité par la discordance qu'il perçoit entre les deux voix, discordance qui l'empêche d'attribuer tout l'énoncé à une seule instance énonciative, comme dans (1), où "c'est la distanciation légèrement moqueuse entre le narrateur et son personnage qui indique la présence d'un fragment de DIL"5 :

(1) Une faculté extraordinaire, dont il ne savait pas l'objet, lui était venue. Il se demanda, sérieusement, s'il serait un grand peintre ou un grand poète ; et il se décida pour la peinture, car les exigences de ce métier le rapprocheraient de Mme. Arnoux. Il avait trouvé sa vocation! Le but de son existence était clair maintenant, et l'avenir infaillible! (Flaubert, L'éducation sentimentale)

Dans d'autres cas, la discordance pourra être perçue grâce à la présence de termes ou de tournures syntaxiques qui n'appartiennent pas au registre du narrateur, comme dans (2) :

(2) - Gaspard ! Eh là, Gaspard ! Où qu'il se cache c'fourneau-là ?... Et en attendant qu'il parût, ils se présentèrent quinze pour faire son portrait et conter ses exploits. Voilà : un type crevant, avec un blair su'l'côté. Une balle crevante. Il était dans le même wagon comme eux : toute la nuit on s'était crevé ! (Benjamin, Gaspard)

D'autre part, son ample éventail de formes énonciatives et syntaxiques fait qu'il constitue un mode de reproduction auquel on peut avoir recours aussi bien pour les reproduc-

5 D. MAINGUENEAU (1986) : Éléments de linguistique pour le texte littéraire, Paris : Bordas, p. 97. 
tions les plus manifestes que pour celles où les frontières entre discours citant et discours cité s'effacent. En effet, le DIL est un phénomène d'hétérogénéité énonciative qui, à la différence du DD et du DI, ne présente pas de marques linguistiques ou typographiques claires, ce qui fait que parfois il ne soit pas nettement différencié du contexte et se confonde avec le discours du narrateur. Souvent, d'un niveau à l'autre, il n'y a pas rupture mais continuité. Hors contexte, rien ne peut conférer de façon certaine à un énoncé le statut de DIL. Et ceci en raison de la propriété qu'il a de rapporter des propos tout en laissant entendre, intimement mêlées, deux voix différentes, deux énonciateurs : l'énoncé ne peut être entièrement attribué ni au narrateur ni au personnage, il n'est pas possible de séparer ce qui provient de l'un et de l'autre. Il est facile de trouver, par conséquent, des cas ambigus au moment de délimiter ses frontières avec précision ou même de déterminer son existence. Le DIL se présente, de cette manière, comme un procédé plus complexe et en même temps plus puissant que les deux autres modes de reproduction.

Bien que le DIL ne possède pas de mode d'introduction spécifique, nous trouvons souvent des indices de sa présence comme l'apparition d'un verbe introducteur en incise ou postposé, qui confirme l'interprétation que l'on doit en faire :

(3) Avant qu'elle se mariât, elle avait cru avoir de l'amour ; mais le bonheur qui aurait dû résulter de cet amour n'étant pas venu, il fallait qu'elle se fût trompée, songeait-elle. (Flaubert, Madame Bovary)

Quelquefois, si nous supprimons ce verbe, l'ambiguïté apparaît immédiatement, comme dans (4) : si l'incise n'apparaissait pas, la relative serait un énoncé primaire, commentaire du narrateur anonyme. La présence de l'incise attribue ce commentaire au locuteur cité, "le commandant", et transforme par là l'énoncé en citation :

(4) Le commandant menaça de sa surveillance et de sa colère ces Européens sans aveu qui venaient, disait-il, s'établir dans le Nouveau Monde. (Nachez. Cité par Bally).

Le DIL le plus intéressant et le plus riche, du point de vue des effets littéraires et des ambiguïtés qui concernent 
l'origine et le support des opérations énonciatives, est sans aucun doute le DIL sans verbe introducteur. Ce qui permet l'identification de ce genre d'énoncés, c'est la relation qu'ils entretiennent avec l'énoncé primaire. En effet, il peut apparaître des pistes dans le cotexte immédiat qui nous avertissent de la présence de la citation : dans ces cas, le DIL fonctionne normalement en tant qu'apposition ou expansion sémantique d'un mot ou d'une expression de l'énoncé primaire, développant ce que celle-ci indique de façon sommaire :

(5) Désespéré de le voir si pâle, le caporal lui demanda paternellement: Ça ne va donc toujours pas, hein ? Est-ce que c'est ton pied encore ? Maurice dit non, de la tête. Son pied allait tout à fait mieux, dans les larges souliers. (Zola, La Débâcle)

Parfois, il n'y a aucun mot concret auquel nous renvoie le DIL. C'est un sentiment absolument indéfini, dilué dans le cotexte, qui nous fait découvrir sa présence, comme dans (6), où rien dans le texte n'annonce les réflexions suivantes :

(6) La maison de Dieppe se trouva vermoulue d'hypothèques jusque dans ses pilotis ; ce qu'elle avait mis chez le notaire, Dieu seul le savait, et la part de barque n'excéda point mille écus. Elle avait donc menti, la bonne dame! (Flaubert, Madame Bovary).

D'autres fois, le DIL apparaît suivi de quelque marque qui confirme sa présence a posteriori, indiquant explicitement l'interprétation que l'on doit en faire, comme dans (7):

(7) Il la retint à déjeuner. Elle attendait sa blanchisseuse, elle devait être rentrée de bonne heure. Cette réponse l'exaspéra. (Huysmans, Marthe)

Quoi qu'il en soit, la structure syntaxique ne traduit pas le changement de niveau énonciatif. C'est ce qui confère au DIL son ambiguïté, ambiguïté qui s'avère tout spécialement évidente lorsque l'on tente de préciser les supports et les origines des expressions référentielles :

(8) Madame Benoît s'y prit adroitement en s'informant de son oncle. Comment allait ce bon parent? (Flaubert, L'éducation sentimentale) 
Ainsi, dans l'exemple (8), l'expression «ce bon parent » ne peut être totalement attribuée à Mme Benoît, surtout à cause du démonstratif « $c e »$. En s'adressant à Frédéric, elle aurait plutôt dit "Comment va votre bon parent?". Cependant, l'adjectif "bon » est un élément affectif qui démontre une bienveillance uniquement attribuable à Mme Benoît. L'ensemble de l'expression est donc mixte. Ceci confirme l'hypothèse que les énoncés au style indirect libre sont des énoncés spécialement polyphoniques : les termes par lesquels le contenu représenté est exprimé peuvent provenir du personnage en question, du narrateur primaire ou de n'importe quel autre personnage du roman.

En effet, dans les énoncés où il y a absence de marques introductrices et où le changement de narrateur ne se reflète pas dans la syntaxe, il est difficile d'établir si le support de l'énoncé est le locuteur cité, le narrateur ou tous les deux à la fois. C'est ce qui arrive dans l'exemple (9): Qui parle dans ce cas ? Le narrateur, Shelley ou l'ami de Southey?

(9) Chez le duc de Norfolk, Shelley avait rencontré un ami de Southey qui lui avait offert de l'emmener chez le poète. Ainsi, pour la première fois, il allait voir en chair et en os un écrivain qu'il admirait. (Maurois, Ariel)

Quelquefois, on trouve dans un même énoncé des signes contradictoires. Ainsi, nous trouvons des énoncés au passé simple - ce qui nous empêche de les considérer comme des DIL $^{6}$-, qui présentent pourtant une modalité qui n'est attribuable qu'à un personnage déterminé. Dans ces cas, nous avons choisi de les considérer comme des énoncés primaires mimétiques, imputables au narrateur, indiquant ainsi que, contrairement à ce qui se passe dans le DIL, nous n'assistons pas à un changement de niveau énonciatif. Ce qui intervien-

6 Tout énoncé au passé simple est perçu, en effet, comme énoncé du narrateur. Il n'y a pas déploiement spatio-temporel de la conscience du personnage - comme il arrive avec l'usage de l'imparfait (voir note 4) -, car rien ne nous avertit de la présence d'une autre situation d'énonciation. Le passé simple, par sa force assertorique et sa position catégorique, porte le procès à l'effectuation, le faisant ainsi échapper à l'intériorité du personnage (cf. M. Hirsch (1980): "Le style indirect libre: linguistique ou histoire littéraire ? ", in A. Joly (éd.) : $L a$ psychomécanique et les théories de l'énonciation, Lille : P.U.L., 79-89). 
drait ici, c'est la focalisation interne, qui fait qu'un énoncé primaire soit très proche d'un énoncé rapporté au style indirect libre. C'est le cas de (10) :

(10) Et cependant, de toute cette fortune que l'on avait fait sonner si haut, rien, si ce n'est un peu de mobilier et quelques nippes, n'avait paru dans le ménage. «Il fallut tirer la chose au clair. » (Flaubert, Madame Bovary).

Conscients de la difficulté qu'implique dissocier la question «qui parle ? » de la question «qui perçoit? », nous défendons cependant que pour représenter le point de vue de l'autre il n'est pas nécessaire d'adopter son discours. De cette façon, bien que tout texte polyphonique soit nécessairement "polyperspectif », le contraire n'est pas obligé, comme certains le pensent ${ }^{7}$. La grammaire possède ses moyens pour rendre perceptible la confluence de voix ; s'il n'y a pas d'indication de convergence, mais seulement un déplacement du foyer, nous nous trouvons en présence du discours du narrateur et non pas en présence d'une reproduction discursive. Nous proposons, par conséquent, de ne pas confondre ce genre d'énoncés focalisés, mais appartenant au discours du narrateur, avec les énonciations rapportées au style indirect libre, parfaitement reconnaissables, en principe, d'un point de vue énonciatif, même si les marques peuvent apparaître neutralisées dans certains contextes. De cette manière, nous tentons de préserver une ambiguïté que nous croyons délibérée.

Quoi qu'il en soit, par nature, le DIL a tendance à estomper ses limites, ce qui le rend difficilement compatible avec des modes d'introduction nettement marqués. Son intérêt est précisément d'atténuer la différenciation entre les deux niveaux : discours citant et discours cité. Les mots ou les sentiments du personnage sont évoqués sans rompre la trame narrative. En effet, nombreux sont les textes dans lesquels nous observons le passage, sans aucune transition et de façon répétée, de la narration au DIL. Parfois, uniquement une syntaxe caractéristique de la langue orale, non imputable au

${ }^{7}$ C'est le cas de M. Bakhtine, pour qui polyphonie est synonyme de polyperspective ou multiplicité de points de vue (cf. The dialogic Imagination, Austin: University of Texas Press, 1981). 
narrateur, ou les expressions propres d'un personnage déterminé nous permettent de le découvrir, comme il arrive dans (11):

(11) Mélanie, au matin, crut sa maîtresse malade et parla d'aller chercher un médecin. Madame n'avait pas dîné la veille; Madame, avec ses traits tirés, était méconnaissable. Mais Élise refusa tout secours médical. (Boylesve, Elise)

D'autre part, en relation avec le DI, le DIL présente l'avantage de pouvoir dépasser amplement le cadre de la phrase, de "construire des unités transphrastiques qui ne soient pas soumises à une lourde armature de dépendances syntaxiques, incompatibles avec la spontanéité et la fluidité des sentiments ou des paroles que le texte rapporte"8, comme nous pouvons observer dans (12) :

(12) Virginie ajouta de nouveaux détails sur elle et son mari : il avait fait un petit héritage, d'une tante; il l'établirait sans doute plus tard; pour le moment, elle continuait à s'occuper de couture, elle bâclait une robe par-ci par-là. Enfin, au bout d'une grosse demi-heure, la blanchisseuse voulut partir. (Zola, l'Assommoir)

Ainsi que nous avons eu l'occasion de le constater, étant donné son statut mixte, le DIL présente, selon les cas, des proportions variables de traits linguistiques caractéristiques du DD et du DI. Cependant, il obéit à certaines contraintes fondamentales destinées à préserver sa spécificité face à ces deux manifestations. En effet, le fait qu'il ne possède pas de marques spécifiques n'implique pas qu'il ne se trouve soumis à aucune règle. Ainsi, dans le DIL sont exclus les éléments qui l'assimileraient totalement au DI et au DD : la subordination à un verbe de locution - ce qui le différencie du DI - et la présence de déictiques personnels ancrés dans la situation d'énonciation du locuteur cité - caractéristique du DD. En effet, les expressions déictiques personnelles qui apparaissent dans l'énoncé rapporté au style indirect libre ne peuvent jamais avoir le locuteur rapporté comme origine. Ainsi, s'il

8 D. MAINGUENEAU (1981): Approche de l'énonciation en linguistique française, Paris : Hachette, p. 114. 
apparaît des déictiques de la 1 ère et de la 2 ème personne, ils ne pourront pas représenter les interlocuteurs du discours cité mais ceux du discours citant. C'est le cas de (13), où les déictiques du DIL ne se comprennent que s'ils sont en relation avec l'énonciation primaire : en effet, ils coïncident avec le «je » du narrateur homodiégétique :

(13) Elle me raconta ce qu'elle avait souffert en essayant de s'éloigner de moi ; que de fois elle avait espéré que je la découvrirais malgré ses efforts, comment le moindre bruit qui frappait ses oreilles lui paraissait annoncer mon arrivée (B. Constant, Adolphe)

À part ces minimes contraintes, le DIL présente, ainsi que nous l'avons vu tout au long de ces pages, des aspects très divers, "oscillant entre ces deux pôles extrêmes que sont, d'un côté, le discours dépourvu des marques de subjectivité du locuteur cité", et pour cette raison voisin du DI, et "de l'autre, un discours proche du $\mathrm{DD}$, où la voix du personnage domine largement celle du narrateur"9, et où apparaissent des manifestations explicites de sa subjectivité.

Ainsi donc, le DIL constitue une technique relativement complexe, utilisée selon les œuvres, les époques et les genres de textes, pour répondre à des nécessités structurales précises, et qui suppose de la part du récepteur une lecture réflexive et une décodification attentive. Pour qu'un discours se comprenne sans ambiguïté en tant que discours rapporté, il doit contenir quelque signe formel qui mette en évidence la mimesis ou représentation d'un autre discours. Dans le DIL, cette mimesis se trouve au niveau des catégories spatiales et temporelles du personnage, en plus de celle concernant les traits expressifs. Quand la superposition des deux énonciations - celle du narrateur et celle du personnage - se manifeste en surface, l'identification d'un DIL est une tâche facile, mais lorsque les marques grammaticales se neutralisent, la condition ambiguë du DIL, qui fait dépendre son identification du contexte et non seulement de traits grammaticaux, est mise en évidence. La fusion ou superposition d'énonciations devient confusion et c'est au lecteur de dissiper l'incertitude.

${ }^{9}$ D. MAingueneau (1986) : op. cit., p. 99. 